\title{
Utility of Fluid Assessment Based on the Intrathoracic Impedance Monitoring in a Peripartum Woman with Heart Disease
}

\author{
Atsushi Daimon, ${ }^{1}$ MD, Chizuko A. Kamiya, ${ }^{2}$ MD, Masami Sawada, ${ }^{2}$ MD, Yusuke Ueda, ${ }^{2}$ MD, \\ Chinami Horiuchi, ${ }^{2} \mathrm{MD}$, Takekazu Miyoshi, ${ }^{2} \mathrm{MD}$, Mitsuhiro Tsuritani, ${ }^{2} \mathrm{MD}$, \\ Naoko Iwanaga, ${ }^{2} \mathrm{MD}$, Reiko Neki, ${ }^{2} \mathrm{MD}$, Hideo Okamura, ${ }^{3} \mathrm{MD}$, \\ Shingo Kusano, ${ }^{3} \mathrm{MD}$ and Jun Yoshimatsu, ${ }^{2} \mathrm{MD}$
}

\begin{abstract}
Summary
Recently, implantable cardioverter-defibrillators (ICD) have become capable of monitoring intrathoracic impedance to detect an increased fluid volume and heart failure. Pregnancy is a well-known cause of an increased body fluid volume; however, it is not clear whether the measurement of intrathoracic impedance by ICD is clinically useful for precisely detecting heart failure in pregnant women. We herein report the case of a 39-yearold woman with an ICD that had been implanted after an event of ventricular fibrillation due to severe aortic regurgitation with a bicuspid aortic valve. Elevated right ventricular pressure and brain natriuretic peptide levels were detected at 37 weeks of gestation and postpartum. At the same time, the ICD's stored fluid index gradually increased and exceeded the threshold on the 10th day after delivery. She was treated with diuretics and recovered from postpartum heart failure. The physiological volume changed in the perinatal period, but we were still able to detect heart failure by ICD. Intrathoracic impedance monitoring is effective in the perinatal field.
\end{abstract}

(Int Heart J 2018; 59: 435-438)

Key words: Pregnancy, Implantable cardioverter-defibrillators, Congenital heart diseases

$\mathrm{N}$ ew medical and device-based therapies for heart failure have improved survival and reduced hospitalization rates. ${ }^{1)}$ Implantable cardioverterdefibrillators (ICDs) or cardiac resynchronization therapy defibrillators (CRT-Ds) have improved survival in patients with life-threatening arrhythmias. Additionally, ICDs and CRT-Ds have the capability of performing automatic physiological and technical monitoring. The number of women with ICDs or CRT-Ds who reach a reproductive age continues to increase, and in recent years, the majority of pregnant women with ICDs have been able to complete and tolerate pregnancy without serious complications. ${ }^{2,3)}$

Pregnancy is associated with several physiological changes to the cardiovascular system, including an increased blood volume, heart rate, and cardiac output and decreased systemic vascular resistance. In some instances, these cardiovascular changes may trigger maternal cardiac deterioration during pregnancy. Clearly, pregnant patients with ICDs require special medical caution with regard to the cardiac function and arrhythmias. ${ }^{2,4)}$

The early detection of worsening heart failure by monitoring an implanted device could enable pre-emptive medical intervention and improve outcomes; however, the evidence of its clinical benefit remains unclear,") especially in pregnant women. Kramer and Maisel reported that a woman with arrhythmogenic right ventricular cardiomyopathy and an ICD capable of monitoring intrathoracic impedance demonstrated a dramatic increase in her Opti$\mathrm{Vol}^{\mathrm{TM}}$ Fluid index levels in the absence of clinical heart failure. ${ }^{6)}$ They concluded that the physiological context of pregnancy limited the utility of intrathoracic impedance monitoring. In contrast, our present study reports that intrathoracic impedance monitoring using an ICD detected precise heart failure in the peripartum period in a patient with cardiac diastolic dysfunction.

\section{Case Report}

A 39-year-old Japanese woman visited our hospital for her third pregnancy. She had been diagnosed with coarctation of the aorta $(\mathrm{CoA})$ and patent ductus arteriosus (PDA) at 8 months of age. At 2 years of age, she underwent PDA ligation and CoA repair. At 25 years of age, she was diagnosed with a bicuspid aortic valve with severe aortic regurgitation and mild aortic stenosis by cardiac catheterization. Because her left ventricle was dilated and its contraction was decreased, she was offered surgical valve replacement, but refused. At 30 years of age, her Holter monitor detected non-sustained ventricular tachy-

From the ${ }^{1}$ Department of Obstetrics and Gynecology, Osaka Medical College, Takatsuki, Japan, ${ }^{2}$ Department of Perinatology and Gynecology, National Cerebral and Cardiovascular Center, Suita, Japan and ${ }^{3}$ Cardiovascular Department, National Cerebral and Cardiovascular Center, Suita, Japan.

Address for correspondence: Atsushi Daimon, MD, Department of Obstetrics and Gynecology, Osaka Medical College, 2-7 Daigakumachi, Takatsuki, Osaka, 569-8686, Japan. E-mail: gyn146@ osaka-med.ac.jp

Received for publication February 17, 2017. Revised and accepted May 1, 2017

Released in advance online on J-STAGE February 14, 2018.

doi: 10.1536/ihj.17-096

All rights reserved by the International Heart Journal Association. 


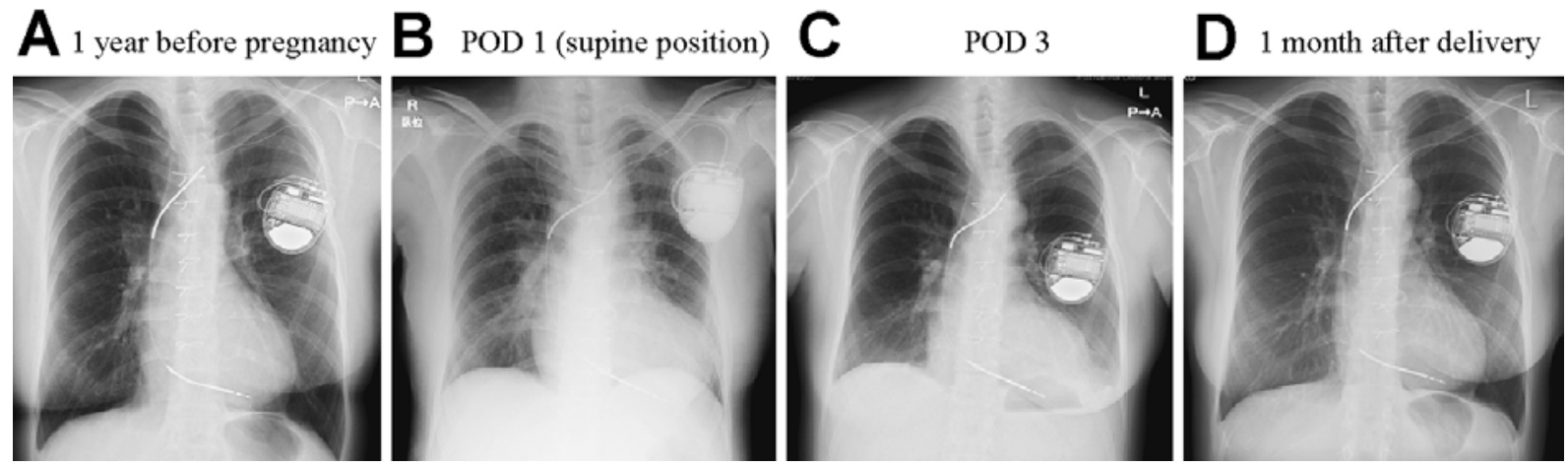

Figure 1. The patient's chest X-ray images. The patient's chest X-ray at 1 year before pregnancy (A, CTR 52\%), POD 1 (B, CTR 67\%), POD 3 (C, CTR 58\%) and 1 month after delivery (D, CTR 52\%). CTR indicates cardiothoracic ratio; and POD, postoperative day.

cardia (NSVT). One month later, she suffered cardiopulmonary arrest with ventricular fibrillation and was resuscitated by electrical defibrillation. She then started medical therapy with $\beta$-blockers and mexiletine and decided to undergo surgery. She underwent aortic valve replacement with a biological valve and ICD implantation. She had been well since the treatments. There was no proper work for arrhythmia in her ICD. She gave birth twice by Cesarean section, due to obstetric reasons, at 32 and 34 years of age. At 36 years of age, her ICD was replaced with an Evera XT ICD VR (Medtronic, Minneapolis, MN, USA) because the battery was running out.

Echocardiography during the early gestational period showed a well-preserved valve function and an improved cardiac systolic function with a left ventricular ejection fraction (LVEF) of 58\%. However, diastolic dysfunction remained; the peak early diastolic left ventricular filling velocity/peak atrial filling velocity ratio (E/A) was 1.8 , the deceleration time was 268 milliseconds, and the peak early diastolic left ventricular filling velocity/peak early diastolic velocity of the mitral annulus (E/e') was 17.6.

At 27 weeks of gestation, a Holter monitor recorded NSVT. She did not have any significant symptoms, and no evidence of heart failure was observed in the physical examinations that were performed at each visit. At 36 weeks of gestation, her brain natriuretic peptide (BNP) level showed an upward trend, and echocardiography showed preserved left ventricular contraction (LVEF $58 \%$ ), and mild pulmonary hypertension (tricuspid regurgitation pressure gradient [TRPG]: $35 \mathrm{mmHg}$ ) due to diastolic dysfunction, which developed in response to volume overload. A week later, at 37 weeks and 2 days of gestation, she was admitted for a repeat Cesarean section. The values of intrathoracic impedance did not exceed the threshold at that time. However, echocardiography showed a more elevated TRPG of $45 \mathrm{mmHg}$, and we administered diuretics (furosemide). The Cesarean section was performed under spiral and epidural anesthesia at 37 weeks and 6 days of gestation, and an infant weighing 2,548 $\mathrm{g}$ was delivered. The total amount of bleeding was $540 \mathrm{~mL}$. At postoperative day 1 , the cardiothoracic ratio, as determined by chest X-ray (decubitus), was $67 \%$, which was markedly larger than the previous value (Figure 1A, B). She started taking diuretics, including furosemide and spi- ronolactone, and a chest X-ray showed dramatic improvement three days later (Figure 1C). After discontinuation of diuretics, she regained weight with worsening bilateral peripheral edema, and her BNP level was increased. Diuretics were therefore restarted. At postoperative day 8, pulmonary hypertension remained (TRPG: $43 \mathrm{mmHg}$ ). Moreover, her blood pressure was elevated, and a calcium antagonist was added. At postoperative day 9, she was discharged with mexiletine, diuretics, and a calcium antagonist.

During her clinical course, ICD device interrogation was performed several times and its function was normal. The patient's volume status over the previous 12 weeks, as indicated by the ICD's stored OptiVol ${ }^{\mathrm{TM}}$ Fluid Index, as well as the thoracic impedance, is displayed in Figure 2. The thoracic impedance began to decrease prior to the delivery (red arrow), and the OptiVol ${ }^{\mathrm{TM}}$ Fluid Index exceeded the alarm threshold at postoperative day 3. The thoracic impedance immediately increased with the start of diuretic therapy (blue arrow).

\section{Discussion}

In the present case, the patient developed heart failure due to diastolic dysfunction, which occurred in response to volume overload and elevated blood pressure in the postpartum period. Heart failure was relieved by the administration of diuretics for the puerperium. The patient's heart failure was monitored by the measurement of body weight, chest X-rays, echocardiography, BNP and by monitoring the thoracic impedance.

The findings from the present case showed that automated detection of a decrease in intrathoracic impedance could provide advanced warning of decompensated heart failure. Furthermore, chest X-rays and echocardiography can only be examined in a hospital, whereas monitoring of the thoracic impedance can be performed at home. The activation of an alarm, such as in the present case, indicates the need for an urgent medical examination.

Despite numerous therapeutic advances, patients with heart failure have a high risk of mortality and morbidity. The ability to predict the patients who will subsequently be hospitalized due to heart failure is limited. Traditional measures of evaluation, including physical signs and 


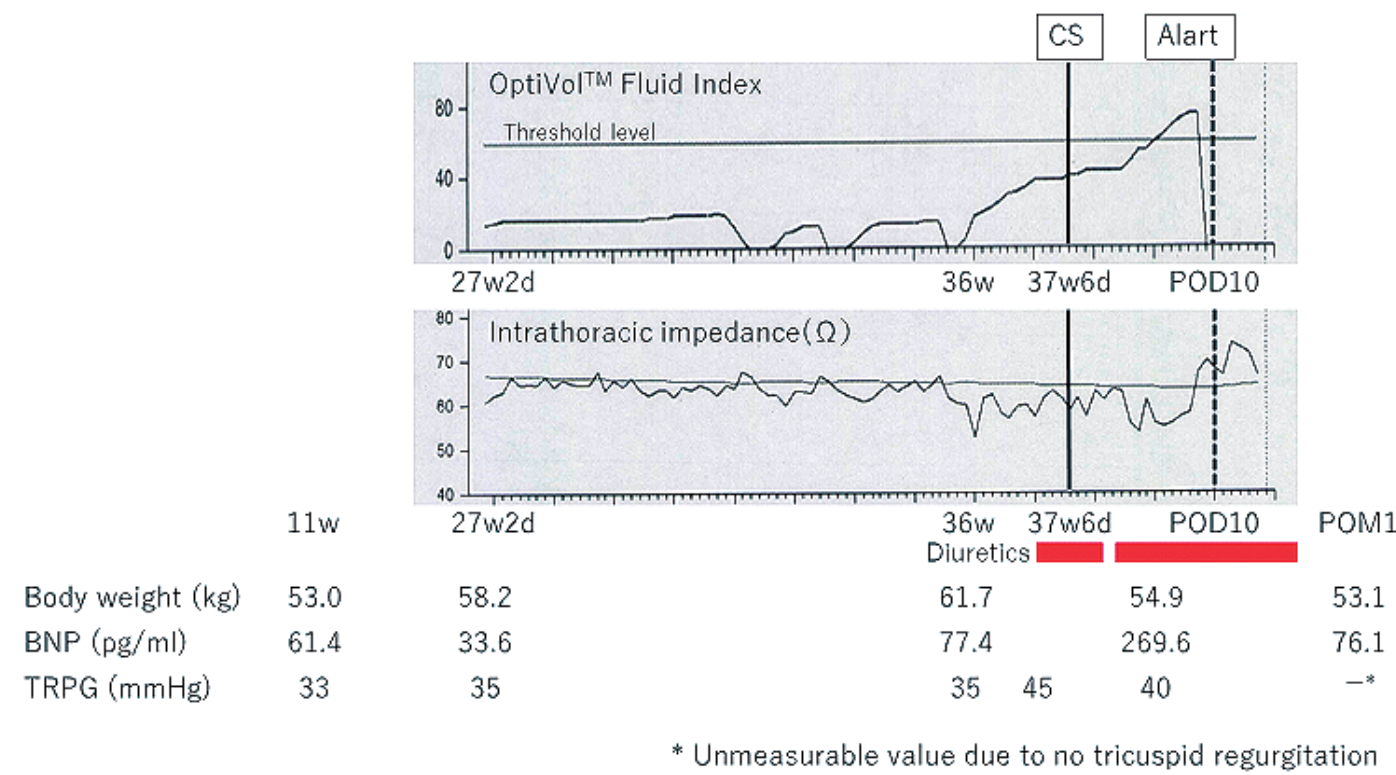

Figure 2. The patient's OptiVol ${ }^{\mathrm{TM}}$ Fluid Index and thoracic impedance during the third trimester and postpartum day 10 , and the patient's physical examinations at each visit. The patient's BNP levels, TRPG by echocardiography, and BW from the 11 th week of gestation to 1 month after delivery. BNP indicates brain natriuretic peptide; POD, postoperative day; TRPG, tricuspid regurgitation pressure gradient; and BW, body weight.

symptoms, are poorly associated with hemodynamics. ICD and CRT-D are recommended by the practice guidelines as a class I therapy for a large number of heart failure patients, based on a clinical trial that showed evidence that their use was associated with a significant reduction in hospitalization and mortality. Intrathoracic impedance, which is measured between the right ventricular lead and an implanted device, is inversely correlated with the pulmonary capillary wedge pressure and the loss of body fluid. Intrathoracic impedance monitoring has the potential to be an alternative indicator of body fluid overload. ${ }^{7)}$ The ambulatory measurement of intrathoracic impedance with an implanted device, including an ICD or CRT-D, may detect increases in pulmonary fluid retention at an early stage. ${ }^{8)}$ It was shown that the diagnostic data from such devices could independently predict hospitalization for heart failure in patients with pulmonary symptoms. The relationship between intrathoracic impedance and changes in the fluid status has been proven by studies that showed a significant correlation between the impedance measurements, the N-terminal pro-B-type natriuretic peptide concentration and the pulmonary capillary wedge pressure. ${ }^{9)}$ Medtronic's proprietary algorithm uses the measured intrathoracic impedance and the patient's reference impedance to calculate the OptiVol ${ }^{\mathrm{TM}}$ Fluid Index. This incorporates the duration and magnitude of daily impedance variations. Recently, the chronic monitoring of intrathoracic impedance using ICD and CRT devices has been proposed as an adjunctive remote monitoring tool to monitor heart failure. The utility of OptiVol ${ }^{\mathrm{TM}}$ Fluid Index has been shown in some studies. The identification of the frequent or extended crossing of the intrathoracic impedance threshold during follow-up may reflect a high risk for the development of adverse clinical events, such as hospitalization. ${ }^{10)}$ The relative sensitivity and rate of detecting unexplained changes in intrathoracic impedance were much higher (76\% versus $23 \%$ ) and lower (1.9 versus 4.3$)$, respectively, in comparison to standard heart failure monitoring using daily weight changes. Intrathoracic impedance monitoring represents a useful adjunctive clinical tool for managing heart failure patients with implanted devices. ${ }^{11)}$ The maternal internal fluid volume is known to exhibit marked fluctuation in the peripartum and lactation periods. The impedance meter is thought to be useful for perinatal body fluid management and to be reliable for monitoring the internal fluid volume.

Several limitations associated with this case report should be noted. First, no reference value of the intrathoracic impedance during the postpartum period has been established. Second, the sensitivity of intrathoracic impedance in detecting heart failure is unknown.

In conclusion, the use of an external impedance cardiac output flowmeter could become a tool that may be noninvasively applied to detect and quantify increases and decreases in the internal fluid volume in peripartum women with heart disease.

\section{Disclosures}

Conflicts of interest: Kusano $\mathrm{K}$ received lecture fees from Medtronic and Boston Scientific. Okamura $\mathrm{H}$ is a recipient of the Medtronic Japan Fellowship 2014 for Young Japanese Investigators offered by the Japanese Heart Rhythm Society.

\section{References}

1. Yu CM, Wang L, Chau E, et al. Intrathoracic impedance monitoring in patients with heart failure: correlation with fluid status 
and feasibility of early warning preceding hospitalization. Circulation 2005; 112: 841-8.

2. Miyoshi T, Kamiya CA, Katsuragi S, et al. Safety and Efficacy of Implantable Cardioverter- Defibrillator During Pregnancy and After Delivery. Circ J 2013; 77: 1166-70.

3. Natale A, Davidson T, Geiger MJ, Newby K. Implantable Cardioverter-Deibrillators and Pregnancy A Safe Combination? Circulation 1997; 96: 2808-12.

4. Agir A, Bozyel S, Celikyurt U, et al. Arrhythmogenic right ventricular cardiomyopathy in pregnancy. Int Heart J 2014; 55: 372-6.

5. Hindricks G, Taborsky M, Glikson M, et al. Implant-based multiparameter telemonitoring of patients with heart failure (INTIME): a randomised controlled trial. Lancet 2014; 384: 58390.

6. Kramer DB, Maisel WH. An Unusual Cause of Abnormal Intrathoracic Impedance in a Patient with Arrhythmogenic Right Ventricular Cardiomyopathy. PACE 2011; 34: e60-

7. Soga Y, Ando K, Arita T, et al. Efficacy of fluid assessment based on intrathoracic impedance monitoring in patients with systolic heart failure. Circ J 2011; 75: 129-34.

8. Nishii N, Kubo M, Okamoto Y, et al. Decreased Intrathoracic Impedance Associated With OptiVol Alert Can Diagnose Increased B-Type Natriuretic Peptide - MOMOTARO (Monitoring and Management of OptiVol Alert to Reduce Heart Failure Hospitalization) Study-. Circ J 2015; 79: 1315-22.

9. Lüthje L, Vollmann D, Drescher T, et al. Intrathoracic impedance monitoring to detect chronic heart failure deterioration: relationship to changes in NT-proBNP. Eur J Heart Fail 2007; 9: 716-22.

10. Small RS, Wickemeyer W, Germany R, et al. Changes in intrathoracic impedance are associated with subsequent risk of hospitalizations for acute decompensated heart failure: clinical utility of implanted device monitoring without a patient alert. J Cardiac Fail 2009; 15: 475-81.

11. Abraham WT, Compton S, Haas G, et al. Intrathoracic impedance vs daily weight monitoring for predicting worsening heart failure events: results of the Fluid Accumulation Status Trial (FAST). Congest Heart Fail 2011; 17: 51-5. 